\title{
In defence of Aristotelian metaphysics
}

\author{
Tuomas E. Tahko
}

\section{I INTRODUCTION}

When I say that my conception of metaphysics is Aristotelian, or neo-Aristotelian, this has more to do with Aristotle's philosophical methodology than his metaphysics, but, as I see it, the core of this Aristotelian conception of metaphysics is the idea that metaphysics is the first philosophy. In what follows I will attempt to clarify what this conception of metaphysics amounts to in the context of recent discussion on the methodology of metaphysics (e.g. Chalmers et al. 2009, Ladyman and Ross 2007). There is a lot of hostility towards the Aristotelian conception of metaphysics in this literature: for instance, the majority of the contributors to the Metametaphysics anthology by Chalmers et al. assume a rather deflationary approach towards metaphysics. In the process of replying to the criticisms towards Aristotelian metaphysics put forward in recent literature I will also identify some methodological issues concerning the foundations of Aristotelian metaphysics which deserve more attention and ought to be addressed in future research.

In Section 2.2 I will compare the Aristotelian and what could be called a 'Quinean' conception of metaphysics. According to the Quinean approach, the key questions of metaphysics concern the existence of different kinds of things, whereas the Aristotelian approach focuses on the natures or essences of these things. A somewhat different attack towards Aristotelian metaphysics can be found in Ladyman and Ross (2007), who group it under the label of 'neo-scholastic metaphysics' - a term which

This paper was written while I was a Visiting Research Fellow at Durham University; this was made possible by a grant from the Academy of Finland. I would like to express my gratitude to the Academy of Finland, to my colleagues in Durham, and most of all to Jonathan Lowe, the supervisor of my doctoral dissertation at Durham, whose work has done so much by way of developing and defending Aristotelian metaphysics. I would also like to thank Richard Stopford for helpful comments on an earlier draft of the paper. 
they use in a strictly pejorative sense: if metaphysics is not supported by current physics, then it has no value.

In Section 2.3 I will consider the approach emerging from the critique by Ladyman and Ross; it aims to naturalize metaphysics. I contend that the call for naturalization is deeply mistaken: not only is Aristotelian metaphysics already naturalized, it is also a necessary precursor of all scientific activities.

Finally, I will hint towards a programme for a rigorous methodology of Aristotelian metaphysics inspired by E. J. Lowe's (e.g. 1998) work. According to this line of thought, metaphysics is primarily concerned with metaphysical possibility, which is grounded in essence. I will reply to the critique that Ladyman and Ross (2007) developed against this view and suggest that it is fully consistent with science, and that the theoretical work in science is in fact also based on this very methodology.

\subsection{SHOES AND SHIPS, AND SEALING WAX}

The view that the central task of metaphysics is to determine "what there is' was popularized by Quine's well-known paper, 'On What There Is' (I948). But it would be a rather crude simplification to claim that the Quinean conception of metaphysics is simply to list the things that exist: shoes and ships, and sealing wax. Rather, the Quinean metaphysician is interested in ontological commitment, namely, what sorts of things are we committed to in our ontology? Shoes and ships, and sealing wax are not the most interesting types of entities in this regard, but pigs with wings are. Consider the following argument:

(I) The number of winged pigs is zero.

(2) There is such a thing as the number of winged pigs.

(3) Hence, there are numbers.

Now, this argument does not aim to establish the existence of pigs with wings, but rather the existence of numbers. However, if it is valid, the implication is that anything that we quantify over in the manner that numbers are quantified over in this argument can be subjected to a similar argument. This includes winged pigs. Arguments of this type have recently received a lot of attention, unduly, in my opinion. The idea behind the argument is that we can formulate existence questions in terms of the existential quantifier: ' $\exists \mathrm{x}(\mathrm{x}$ is a number)?' (cf. Fine 2009: I57). But if we can settle these existence questions with the help of arguments such as the one above, then metaphysics does not seem like a very interesting endeavour. While some deflationists about metaphysics would 
welcome this result, I am more inclined to think that we are not in fact dealing with a very typical metaphysical question here. Another worry is that if most metaphysical questions are really existence questions of this type, then these questions are not only trivial, but already addressed by special sciences such as mathematics and, in the case of existence questions concerning material objects, physics. It may be of some comfort that many existence questions are obviously non-trivial. For instance, 'Does the Higgs boson exist?' appears to be a very important and non-trivial existence question. Of course, it has nothing to do with metaphysics. This is not to say that metaphysicians should not be interested in existence questions of this type, but they are certainly not what metaphysics is centrally concerned with.

Perhaps this is not a fair reading of what the Quinean tradition takes metaphysics to be about. After all, existence questions such as the one about numbers have a certain ambiguity about them, and even if we formulate them in the manner suggested above, i.e. ' $\exists x(x$ is a number)?, there is still the question of how we should interpret the existential quantifier. We have several options in this regard, but here I will focus on Thomas Hofweber's (2005, 2009) suggestion, according to which we can distinguish between the internal and the external reading of the existential quantifier. ${ }^{1}$ Let me borrow some examples from Hofweber (2009: 276 ff.) to illustrate this distinction. Consider the following statement: 'Everything exists.' The first reaction to this statement is that it is trivial, as naturally everything in the world exists. But then again there would appear to be some things, such as Sherlock Holmes, that do not exist, and hence not everything exists. In this sense, the statement is apparently false. These two senses are supposed to correspond with the external and the internal readings of the existential quantifier, respectively. Essentially, the external reading concerns objects within the domain of the quantifier, whereas the internal reading concerns the inferential role of the quantifier, i.e., the role that the quantifier has in linking quantified statements to quantifier free statements. Hofweber illustrates the distinction with sentences such as 'Someone kicked me', where the natural reading of the quantifier is external since whoever it is that kicked me is surely within the domain of the quantifier 'someone'. But the same quantifier can have a different interpretation in sentences such as 'There is someone we both admire', where the reading is internal when I have forgotten who it is that we both

I will not discuss the differences between the objectual and the substitutional reading of the existential quantifier here, but see Crane (this volume) for further options. 
admire. In this case I just want to say that there is some X that we both admire, but I cannot remember who $\mathrm{X}$ is. A quantifier is needed in this case as well, but we would not want it to range over what the world contains: if it happens to be Sherlock Holmes that we both admire, then X would not be contained in the world at all. So, the internal reading remains neutral about the domain; we want to be able to replace it with any term, whether or not that term refers to something that exists in the world.

This distinction is motivated by Carnap's internal-external distinction, but the historical details are unimportant for my purposes. What Hofweber hopes to cash out with this distinction - and I suspect that many contemporary metaphysicians would be sympathetic to the move is a way to address the apparent problems that existence questions pose for metaphysics. The upshot is that we have two ways to go regarding our talk about a given metaphysical problem, such as the one concerning the existence of numbers: internalism and externalism. Internalism is the view that quantification over things like number terms is non-referential in ordinary uses (it does not aim to refer to some domain of entities) and externalism is the view that it is commonly referential (it refers to a domain of entities). Hofweber (2009: 284) suggests that the question 'Are there numbers?' is underspecified because it has both an internal and an external reading. The argument for the existence of numbers that opened this section relies on the internal reading, and on this reading the existence of numbers seems to be trivially true. However, with an external reading the question is not trivial, and furthermore, Hofweber thinks that mathematics does not provide an answer to the external reading. Accordingly, perhaps it is the external reading of the question that metaphysics is concerned with: it is not trivial, nor is it answered by the special sciences. This is the middle way between esoteric and deflationist metaphysics that Hofweber proposes: metaphysics attempts to settle what there is, but this question is neither trivial nor settled by the special sciences.

So, according to Hofweber, metaphysics is interested in the external reading of questions such as 'Are there numbers?'. But the correct answer to this question still depends on whether internalism or externalism is true about numbers, that is, whether our ordinary talk about numbers is such that it aims to refer to some domain of entities, 'numbers', or does not. The task of metaphysics is now supposed to be to answer this question. In the case of numbers, Hofweber argues that internalism is the most plausible choice, as it seems that talk about numbers does not aim to refer to a particular domain of entities; in this sense numbers words are like the words 'some' and 'many' (2009: 286). Since numbers are non-referential, 
there is nothing in the world that they pick out and hence no such things as numbers. In general, Hofweber suggests that internalism settles many of the problematic external ontological questions, and that the task of metaphysics is merely to decide whether internalism or externalism is true regarding these external questions:

There is no distinct metaphysical method to address ontological questions. To find the answer we have to decide between internalism and externalism, which is done with the methods employed in the study of language, and related issues. (Hofweber 2009: 287)

This is Hofweber's ambitious, yet modest, metaphysics. However, I do not see how it differs from the deflationist approach; by the looks of it, metaphysics turns out to be a rather trivial and uninteresting endeavour, its only task being to determine whether internalism or externalism is true in given cases. In fact, even this task is delegated to the special sciences, since Hofweber states that it is by using the methods 'employed in the study of language, and related issues' that we determine whether internalism or externalism is true (2009: 287). Metaphysics, it turns out, is really nothing but linguistics.

I do not think that this result is particularly surprising. If existence questions are considered to be the core questions of metaphysics, then something has gone wrong to begin with. It is true that numbers, for instance, have received considerable attention in metaphysics, however, the form of the metaphysical question concerning numbers ought not to be whether there in fact are any such things, but rather what is the nature of these things. But what is this other question, the question about the nature of numbers? Well, if we acknowledge that metaphysics does not concern the existence of numbers but we nevertheless think that there are some important and interesting metaphysical questions about numbers, then the questions must concern the status or type of these entities. The way Kit Fine puts this is that the question is about whether numbers are real:

The realist and anti-realist about natural numbers, for example, will most likely take themselves to be disagreeing on the reality of each of the natural numbers $-\mathrm{O}, \mathrm{I}, 2, \ldots$; and this would not be possible unless each of them supposed that there were the numbers $\mathrm{O}, \mathrm{I}, 2, \ldots$ It is only if the existence of these objects is already acknowledged that there can be debate as to whether they are real (Quine's error, we might say to continue the joke, arose from his being unwilling to grasp Plato by the beard). (Fine 2009: 169)

Indeed, Fine suggests that existence should be considered as a predicate: if we wish to ask whether integers exist, we should not formulate the 
question like this: ' $\exists x I x$ ?', where 'I' refers to integers, but rather like this: ' $\forall \mathrm{x}(\mathrm{Ix} \supset \mathrm{Ex})$ ?', where ' $\mathrm{E}$ ' is the predicate for existence - the question is not whether some integer exists, but whether every integer does (Fine 2009: 167). In fact, many existence questions are for the most part only interesting to scientists, or mathematicians in the case of numbers. Accordingly, Fine takes the realism/anti-realism debate to concern the reality of objects, and in order to have a debate about this in the first place realism in the usual sense has to be assumed. The upshot of this line of thought is that the realism/anti-realism debate in the usual sense is a non-starter, because either we are all realists, or there is no discussion to be had.

Although I am very sympathetic to the line that Fine takes, there are some problems that remain to be solved, for we are now on our way towards what Hofweber has scornfully dubbed 'esoteric metaphysics'. The folly of esoteric metaphysics, as Hofweber defines it, is that metaphysical questions should be answered with metaphysical terminology, and an understanding of metaphysics is needed for one to even be able to understand metaphysical questions (Hofweber 2009: 266 ff.). This bears some similarity to the idea that there is a specific 'ontology room' where we can sensibly doubt the existence of tables, or a particular language of metaphysics where questions such as this make sense. The obvious problem with this approach is that it seems particularly mysterious in the light of a metametaphysical analysis: it may save metaphysics, but it does not help in clarifying what metaphysics is about. Indeed, I do not think that this is the way to go. Metaphysics is already mysterious enough for the layman, and to say that we are talking in a 'metaphysical sense' that 'ordinary' people cannot understand when we inquire into the existence of tables will surely be the end of what little funding still finds its way towards the study of metaphysics.

There are more sophisticated versions of so-called 'esoteric' metaphysics however, such as the ones proposed by Kit Fine (200I, 2009, this volume) and Jonathan Schaffer (2009). Central to both of these approaches are certain core concepts such as GROUND and PRIORITY, capitalized to separate them from the ordinary usage of these terms (following Hofweber 2009). I do not wish to go into the details of either view, but both of these approaches hold that a central part of metaphysics is the study of ontological dependence: certain things depend on other things for their existence and identity and metaphysics is interested in these dependency relations (cf. Fine 1995a, Correia 2008). Hofweber's main concern with these approaches is that they take certain notions as primitive and that seems to make metaphysics dangerously inaccessible: 
As far as I understand Fine's view, it is a sophisticated version of esoteric metaphysics: metaphysics is supposed to find out what is GROUNDED in REALITY, in a special metaphysical sense of these terms. To know what this sense is gives you entrance into the discipline, but it takes a metaphysician to know this sense. Esoteric metaphysics never sounded so exclusive. (Hofweber 2009: 270)

After a similar analysis of Schaffer's views, Hofweber concludes as follows:

Esoteric metaphysics appeals to those, I conjecture, who deep down hold that philosophy is the queen of the sciences after all, since it investigates what the world is REALLY like. The sciences only find out what the world is like, but what philosophy finds out is more revealing of reality and what it is REALLY like. (Hofweber 2009: 273)

Hofweber continues to pour scorn on esoteric metaphysics by suggesting that it opens the door to views such as the one familiar from Thales: everything is ultimately water. But this is just a straw man; the real problem is whether metaphysical questions can be formulated in the manner that is commonly assumed in contemporary analytic metaphysics. The answer that Fine offers is a resounding 'no', and I am inclined to agree with him. Still, Hofweber is right to ask for more, as it is true that this type of approach to metaphysics is not particularly well developed. It is, however, difficult to develop a view which is not taken seriously to begin with. Unfortunately, the Quinean interpretation of ontological questions is very deep-rooted in contemporary metaphysics.

The distinction between (neo-)Aristotelian and Quinean metaphysics should now be somewhat clearer, and we have already identified some aspects of Aristotelian metaphysics that need to be developed. But before we attempt to engage with the problems that have been identified so far, let us turn to another critique of Aristotelian metaphysics - potential lines of development will be discussed in the final section.

\subsection{NATURALIZING ARISTOTELIAN METAPHYSICS}

I think that philosophy is indeed the queen of the sciences. However, this in no way entails that one needs to be educated in metaphysics to be able to pursue metaphysical questions or to gain access to metaphysical truths - there is no doubt that it will help, but it is not necessary. Moreover, I do not think that philosophy is the queen of the sciences because it is concerned with what reality is REALLY like: I think that both philosophy and science are concerned with reality in this capitalized 
sense. In fact, this is why I take metaphysics and science to be continuous. It should be emphasized here that when I say that metaphysics and science are continuous, I do not mean it in the sense that they would both have exactly the same agenda. Rather, I mean that we could not really engage in one without the other, that is, we could not get very far in our inquiry into the nature of reality with just one of these disciplines. Because of this, it might be better to say that metaphysics and science complement each other (cf. Lowe 20II).

This idea is compatible with Aristotle's writings about the relationship between the study of 'being qua being', i.e., metaphysics, and the special sciences:

There is a science which investigates being as being and the attributes which belong to this in virtue of its own nature. Now this is not the same as any of the so-called special sciences; for none of these others deals generally with being as being. They cut off a part of being and investigate the attributes of this part - this is what mathematical sciences for instance do. Now since we are seeking the first principles and the highest causes, clearly there must be some thing to which these belong in virtue of its own nature. (Metaphysics I003a22-28)

Thus, Aristotelian metaphysics is the study of being as it is in itself, whereas the special sciences investigate only a part of that being. But how well does this view sit with the modern scientific view? Not very well, if Ladyman and Ross (2007) are right. They begin their book, Every Thing Must Go with a brief critique of metaphysics which sweeps over Aristotle's metaphysics as well as contemporary analytic metaphysics. Their primary criticism is that metaphysics suffers from a lack of scientific rigour and is in fact very badly informed of the latest developments in science. The result is a domestication of certain aspects of contemporary science at best, pseudo-scientific mumbo-jumbo at worst. Instead, Ladyman and Ross call for naturalized metaphysics - metaphysics which is based on science. Can we reconcile Aristotelian metaphysics with this idea of naturalized metaphysics?

The main problem that Ladyman and Ross raise for the prospect of reconciling metaphysics - understood in the Aristotelian sense - with natural science is that this type of 'neo-scholastic metaphysics', as they call it, gives priority to a priori, armchair intuitions, and ignores the fact that recent empirical results show the natural world to be much more complicated than our armchair intuitions might suggest. It is difficult to deny this: if we consider Aristotle's ideas in his Physics for instance, most 
of them seem rather obsolete. No philosopher in Aristotle's time could have realized just how small and strange the world of subatomic particles is, or indeed how vast and old the universe is. Having said that, the manner in which Ladyman and Ross characterize 'neo-scholastic metaphysics' is almost as misinformed about the nature of Aristotelian metaphysics as Aristotle perhaps was about natural science.

Let us start with armchair intuitions. Ladyman and Ross (2007: IO-15) give a number of examples of metaphysical, armchair intuitions which seem to be blatantly incorrect from a scientific point of view. Indeed, it is easy to find such examples, in metaphysics and science alike - just consider the repeatedly stated yet later falsified intuition that we have reached the fundamental level of reality when a new microparticle is found. Intuitions are clearly not very trustworthy, but does this mean that armchair reasoning is completely worthless? Even Ladyman and Ross do not go as far as to claim this, for they admit that it is often said of a good physicist 'that he or she has sound physical intuition' (2007: 15). But the use of the word 'intuition' is supposedly different in this case, as it refers to 'the experienced practitioner's trained ability to see at a glance how their abstract theoretical structure probably - in advance of essential careful checking - maps onto a problem space' (2007: 15). There is an ongoing debate in metaphysics about the nature and role of intuitions (e.g. Booth and Rowbottom forthcoming), but I believe that this description is entirely accurate for the metaphysician's use of the term as well, contrary to what Ladyman and Ross claim. Is it not the case that a metaphysician's intuition is exactly a preliminary judgement about how a certain abstract theoretical structure probably maps onto a problem space?

Ladyman and Ross further distance metaphysicians' intuitions from those of scientists by pointing out that the former are often taken as evidence whereas the latter are only heuristically valuable, but this is certainly not a commonly accepted view. In fact, my own view is that intuitions are, for the most part, misleading, exactly because the problem space is generally much more complicated than one first assumes. Regardless, certain very experienced practitioners both in science and in metaphysics may use intuitions as a good heuristic tool. The intuitions themselves are at best only prima facie evidence: careful study, or in some cases empirical research, is required before they can be accepted. But it is certainly a naïve view of metaphysics to assume that metaphysicians simply take their intuitions at face value and leave it at that - generally a book-length study follows! 
Since intuitions are a controversial subject in any case, perhaps we would be better off talking about a priori inquiry in general, as most proponents of (neo-)Aristotelian metaphysics at least contend that some kind of a priori inquiry is possible. One account about the role of this inquiry comes from Lowe (1998), who suggests that metaphysics studies the realm of metaphysical possibility - the space of the possible fundamental structures of reality - but we also need empirical science to determine which of the possible structures corresponds with the actual world. This is one way to understand the Aristotelian conception of metaphysics: the first philosophy studies the fundamental structure of reality, possibility strictly in virtue of being qua being, but we need the second philosophy, the special sciences, to determine how this structure is reflected in the actual world. Ladyman and Ross are aware of this general understanding of metaphysics and in fact claim to endorse the idea that the goal of metaphysics is to unify the special sciences, but:

[W] differ with Lowe on how this task is to be accomplished, because we deny that a priori inquiry can reveal what is metaphysically possible. Philosophers have often regarded as impossible states of affairs that science has come to entertain. For example, metaphysicians confidently pronounced that non-Euclidean geometry is impossible as a model of physical space, that it is impossible that there not be deterministic causation, that non-absolute time is impossible, and so on. Physicists learned to be comfortable with each of these ideas, along with others that confound the expectations of common sense more profoundly. (Ladyman and Ross 2007: 16-17)

This is all that Ladyman and Ross say against the possibility of a priori inquiry into metaphysical possibility, so it is this critique that we must repel if we hope to defend Aristotelian metaphysics. However, I think that what we have here is a very uncharitable interpretation of Lowe's conception of the methodology of metaphysics. This is because Ladyman and Ross seem to assume that our epistemic access to metaphysical possibility has to be infallible. While the infallibility of a priori inquiry may have been a doctrine of Cartesian metaphysics, it is most certainly not a doctrine of Aristotelian metaphysics. Hence, it is true that metaphysicians, like scientists, make mistakes. Kant held that non-Euclidean geometry is impossible, but physics soon showed that not only is it possible, but actual. In fact, in non-Euclidean geometry we have a good case study about how a priori inquiry can indeed reveal what is metaphysically possible. For if we look at the historical facts, it was not empirical inquiry that revealed the possibility of non-Euclidean geometry, but mathematical 
and thus, we might argue, a priori inquiry: the mathematicians Gauss, Lobachevski, and Riemann developed alternative, non-Euclidean geometries which replaced the controversial parallel postulate of Euclidean geometry with an alternative axiom. This way we get a number of possible geometries, although we know that only one of them can be actual. Kant did indeed make a mistake, but the mistake was not due to a flaw in the methodology of a priori inquiry, but rather a failure to grasp the possibility of alternative geometries. The lesson that we should take from this is that the space of metaphysical possibilities reached by a priori means is revisable, quite similarly to empirical results which can be revised in the light of new empirical data.

In fact, the methodological similarities between science and metaphysics are much greater than this. Recall the discussion about intuitions above. As we saw, Ladyman and Ross acknowledge that something like intuitions are used in science as well, although merely as a heuristic tool. Let us imagine a situation where a scientist uses her intuition to come up with a scenario that might explain some empirical data, a new model about, say, gravity. The first thing that she is likely to do is to formulate the model suggested by her intuition with mathematical rigour, which will enable her to determine whether the model is consistent. Sometimes models based on such 'hunches' turn out to be inconsistent, but if this is the case, the error can be spotted early on, certainly before any empirical tests need to be performed. So far, this story is not very far from how a metaphysician would proceed. The metaphysician is perhaps unlikely to use mathematics to model the insight in question, but logic and other means of careful analysis would certainly be in the metaphysician's toolbox. To me, both of these activities seem to be a priori activities, since no empirical elements are present, but I do not want to dwell on the question of whether mathematics is a priori or not. It is sufficient to note that the scientist and the metaphysician proceed from intuitions to detailed models in a similar manner - both use their intuitions merely as a heuristic tool, and both acknowledge the fallibility of this heuristic tool.

It is the next stage of the story which one might expect to demonstrate the superiority of empirical science over armchair metaphysics, namely, the empirical experiments which the scientist can design and use to test the validity of the original intuition. The model, if it is consistent, will only provide proof of the possibility of a correspondence with the actual reality, and only an empirical experiment can reveal which model reflects the actual structure of reality. Of course, this 
empirical element is also available for the metaphysician, but only insofar as her model makes testable predictions. Because metaphysics in the Aristotelian tradition rarely makes such predictions, Ladyman and Ross think that it should not be taken seriously. In fact, they think that the value of metaphysical claims is very limited indeed:

Any new metaphysical claim that is to be taken seriously should be motivated by, and only by, the service it would perform, if true, in showing how two or more specific scientific hypotheses jointly explain more than the sum of what is explained by the two hypotheses taken separately, where a 'scientific hypothesis' is understood as an hypothesis that is taken seriously by institutionally bona fide current science. (Ladyman and Ross 2007: 30)

This 'Principle of Naturalistic Closure' would seem to reduce metaphysics merely to the task of unifying scientific hypotheses. But this ignores a crucial element of the story: metaphysical inquiry is required in order to produce scientific hypotheses in the first place. We have seen that, on the face of it, the process by which metaphysicians and scientists proceed from intuitions to models of reality is rather similar. Specifically, it appears to be non-empirical. Moreover, these models ought to be not just formally consistent, but also consistent with the current empirical data. In the spirit of the Aristotelian tradition, a metaphysician should be familiar with this empirical data - Aristotle certainly seems to have had a good knowledge of the (rather limited) empirical data of his time; in fact he engaged in some empirical research himself, especially in biology. In any case, the core of the matter concerns the nature of the reasoning process which leads to the formulation of a scientific hypothesis. This process is fallible, but it fulfils all the essential elements of metaphysical inquiry. If this is correct, then our attention should not be directed towards the question of whether Aristotelian metaphysics can be naturalized, but rather what the metaphysical foundations of natural science are.

\subsection{THE METHODOLOGY OF ARISTOTELIAN METAPHYSICS}

Although the analysis of the methodology of Aristotelian metaphysics suggested by Ladyman and Ross is flawed in its requirement for infallibilism, it does raise a point that must be addressed. This point concerns the epistemology of metaphysics. Ladyman and Ross (2007: 16) refer to Ted Sider disapprovingly in this connection and point out that Sider's strategy to defend a priori metaphysics by claiming that the epistemological foundations of science and mathematics are equally mysterious is not convincing. Indeed, although I think that there will be an important overlap 
between the epistemological foundations of science and metaphysics, the problem seems to be more pressing for the latter, since the empirical elements of science give it a rather more effective tool to spot errors than anything we have in metaphysics.

So, I acknowledge that there is more work to be done on the foundations of (neo-Aristotelian) metaphysics. Specifically, I think that we need an account of modal epistemology: how can we reliably inquire into the realm of metaphysical possibility given that we sometimes make mistakes? This problem is analogous to a more general debate in modal epistemology, namely the one concerning the link between conceivability and metaphysical possibility (cf. Gendler and Hawthorne 2002). Conceivability arguments are a familiar tool in metaphysics, but one problem is that it seems to be easy to conceive of metaphysically impossible things as well. In any case, it appears that for something to be possible, it must also be conceivable (at least by an ideal conceiver), but although this is a necessary requirement for possibility, it is not sufficient. We seem to have a similar situation with regard to a priori access to metaphysical possibility. In fact, it may appear that these are one and the same problem, since conceivability is sometimes defined in terms of a priori reasoning. I think that this is misleading: it is a short step from conceivability to conceptual analysis, which is another typical way to understand what conceivability means - imaginability in terms of the definitions of concepts - but a priori access to metaphysical possibility cannot be based strictly on conceptual analysis because concepts do not give us access to being qua being; they concern merely a part of being, not the nature of reality in general.

Unfortunately Ladyman and Ross make the mistake of identifying metaphysical a priori inquiry with conceptual analysis. They claim that Lowe follows Frank Jackson and others 'in advocating the familiar methodology of reflecting on our concepts (conceptual analysis)' (2007: 16), and immediately ask how conceptual analysis could possibly reveal anything about the structure of reality. Well, this is a concern that a proponent of Aristotelian metaphysics shares. Moreover, it is the very reason why metaphysical a priori inquiry cannot be identified with conceptual analysis. It should also be noted that far from following Jackson, I believe that Lowe would be as critical of Jackson's project as Ladyman and Ross themselves are. It is a mystery to me where they get the impression that metaphysics in this tradition has anything to do with conceptual analysis.

Nevertheless, we do have to face the problem of modal epistemology. The groundwork for an appropriate account of modality is due to Kit 
Fine's article 'Essence and Modality' (1994), which helped to rejuvenate the Aristotelian idea that the notion of essence is more fundamental than that of modality and that the latter is grounded in the former. Kathrin Koslicki's contribution to this volume also deals with this topic. It is not possible to discuss all the details concerning this issue here, so instead I will attempt to motivate one of the underlying ideas, namely, that possibility precedes actuality. This is effectively what Ladyman and Ross deny, as they are opposed to the idea that metaphysical a priori inquiry could reveal what is possible in advance of empirical research.

The idea of possibility preceding actuality is central especially in Lowe's work, as it forms the basis for the possibility of metaphysics: 'In short, metaphysics itself is possible - indeed necessary - as a form of rational human inquiry because metaphysical possibility is an inescapable determinant of actuality' (Lowe 1998: 9). ${ }^{2}$ So, metaphysics deals with possibilities - metaphysical possibilities - but is not able to determine what is actual without the help of empirical research. However, it is crucial for this account that empirical knowledge in itself is not able to determine what is actual either, for a priori inquiry is needed to delimit the space of possibilities from which the actual structure of reality can be identified by empirical means. Consequently, a priori inquiry is necessary and prior to knowledge about actuality, because without this metaphysical delimitation of what is possible, the space of possibilities would be too vast to handle. So, it is this a priori delimitation of the space of possibilities which enables us to pick out just the genuine metaphysical possibilities from the enormous space of conceivable yet metaphysically impossible things.

Consider the following statement, which is commonly thought to be metaphysically necessary: 'Gold is the element with the atomic number 79.' If this statement is indeed metaphysically necessary, the necessity must be due to the nature of elementhood, that is, the atomic number is, by metaphysical necessity, associated with one and only one element (cf. Tahko 2009b). However, it is certainly conceivable that a different organization of subatomic particles would produce exactly the same characteristics that gold has, and indeed be gold to all ends and purposes. Examples like this are familiar from Putnam's Twin Earth scenarios, but they are generally considered not to entail metaphysical possibility. In any case, there is nothing contradictory in the scenario where the element

2 It should be noted though that what follows is my own conception of the methodology of neoAristotelian metaphysics. It is inspired by the work of Fine and Lowe, but is not necessarily entirely faithful to either. See also the contributions of Fine and Lowe in this volume. 
with atomic number 78 , for instance, has the characteristics that gold has instead of the ones that platinum has. Whether or not this element would be gold is the question that the Twin Earth scenarios are supposed to raise and the correct answer is typically considered to be that it would not be gold. Regardless of this, we can easily imagine a completely different organization of the fundamental physical constants and the laws of physics that would enable this possibility. The usual conclusion is that whatever the resulting element would be, it would not be gold as we know it. At best, it is an epistemic possibility that this element would be gold, but we would be making a metaphysical error if we thought that it could be the same gold that we know and value highly. In fact, there are infinitely many such conceivable scenarios, which are nevertheless metaphysically impossible. Due to our limited rational capabilities, it is impossible for us to consider all of these alternative scenarios - we have to delimit this space of possibilities somehow if we wish to make any progress in science. This is where a priori inquiry is required: the conclusion that the atomic number is a part of the essence of elements is the result of a combination of empirical research and a philosophical, a priori analysis of the different possible ways to interpret the empirical data. ${ }^{3}$

There is more to be said about the relationship between metaphysical a priori inquiry and empirical data. To emphasize that a priori inquiry is needed before empirical data becomes intelligible, let us consider another example, namely the basic thesis of the identity theory: brain states are mental states. This is an a posteriori identity claim and its status, I take it, is currently unsettled. Now, the question is: what sort of empirical information could verify this identity claim? We certainly have ample information about what happens in our brains, yet few physicalists would claim that this is by any means enough to settle the debate. In fact, I think that it is fair to say that no amount of purely empirical information could settle the debate by itself, for otherwise the debate would perhaps be over already. ${ }^{4}$ d do not wish to go into the literature about the 'explanatory gap' here, but the idea is that we lack sufficient information about the underlying a priori identity, namely, we do not know whether this identity holds or not. Note once again though that the understanding

3 Although the atomic number is commonly accepted to be a part of the essence of elements, the issue is not as simple as it might seem; alternative accounts have been suggested. These different accounts may all be consistent and a priori, but only one of them, at most, is actual. See Hendry (2006) for further discussion about elementhood, specifically for one alternative account.

${ }^{4}$ Admittedly, some physicalists especially may think that the debate is indeed over, but given the number of articles and books that continue to be published on the issue, it appears that far from being over, the debate is in fact only heating up. 
of aprioricity at hand here is not synonymous with conceptual analysis this view is familiar from the work of Frank Jackson and others, but from an Aristotelian point of view the identification of a priori inquiry with conceptual analysis is a watered-down conception of the nature of a priori inquiry.

So, although a central theme in the literature on the explanatory gap concerns the role of a priori conceptual analysis which is required to settle the status of the identity theory, this is not the idea that I am advocating. In fact, I think that the role of the a priori part in the mind-brain identity thesis is exactly the same as in the matter-energy (or better: mass-energy) identity thesis, to use an example also familiar from the explanatory gap literature. The a priori work required in the latter case does not concern an analysis of the concepts of 'mass' and 'energy', but rather the natures of mass and energy. Einstein's insight was that really we are only talking about the nature of one thing, namely energy; mass energy, which we observe as matter, is just one of many forms of energy. In the terminology of Aristotelian metaphysics, it would appear to be a part of the essence of energy that it can exist in many forms, one of them being mass energy. Now, it is worth emphasizing here that when Einstein formulated special relativity, one consequence of which is the mass-energy equivalence, he certainly did not do this experimentally, but rather by carefully considering the different possibilities of the behaviour of objects travelling at speeds approaching the speed of light. Much of this work is mathematical, but whether or not we consider mathematics to constitute a priori work, there must be something that this mathematical work is based on as well - perhaps it could be described as an intuition in the sense that was introduced in the previous section.

Returning to the case of mind-brain identity, the upshot would seem to be that even the possibility of mind-brain identity has not been sufficiently characterized, nor has the possibility of mind-brain duality. The stalemate in contemporary philosophy of mind amounts to just this: the a priori delimitation of the different possibilities available in explaining consciousness has not been completed, at least not in sufficient detail to convince the majority of philosophers. Many philosophers are convinced that it is impossible to explain consciousness in terms of the physical, whereas others think that this is the only possible explanation. But since it seems to be very difficult to come to an agreement about which of these possibilities are genuine, the result is that we do not even know what sort of empirical information could verify or falsify the identity claim in question. It is possible that we already possess this empirical information, 
but as the a priori work concerning this debate has not been completed, the empirical information is of little use to us. The same, I think, is true of many other a posteriori identity claims, perhaps even of our previous example about elementhood.

More generally, this methodological picture suggests that the way in which we interpret and analyse empirical information is dependent on an a priori delimitation of what is possible. In some cases the a priori work has been done long ago, whereas some cases seem to elude definite a priori characterization rather effectively. There are plenty of examples of this in science as well: for instance, it appears that no amount of empirical information will settle the most important and most difficult questions concerning quantum mechanics, such as whether the wavefunction has an objective existence or whether it is merely a mathematical convenience, or how the role of the observer should be accounted for in the universal wavefunction, or whether a realist or an anti-realist interpretation of quantum mechanics is correct; any attempt to address issues such as these will have to start from metaphysics.

Some difficult questions about the epistemic role of a priori inquiry remain. From what has been said above, it may still seem that we are dealing with some sort of mysterious rational intuition, since a priori inquiry provides the parameters for any interpretation of empirical data while also being self-correcting. Even given fallibilism, there remains a problem concerning the justification of the criteria used to evaluate a priori propositions. But here as well I believe that Aristotelian metaphysics has a long tradition of research, for the Aristotelian categories are central to the task of determining the criteria by which we judge both empirical and rational information. Several chapters in this volume discuss either the categorical structure of reality in general or specific categories; this is exactly the type of research needed to examine the foundations of metaphysics. It should be emphasized though that this research goes hand in hand with empirical research: there is a bootstrapping relationship between a priori and a posteriori inquiry, and we cannot engage in one without the other (Tahko 2008). Certain a priori principles, such as the ones emerging from the categorical structure of reality, may be more fundamental, and it is perhaps with these principles that the bootstrapping begins. One of the obvious candidates for such a fundamental principle - one that Aristotle certainly considered to be fundamental - is the law of non-contradiction. Perhaps the law of non-contradiction could be self-evident enough to act as a foundational a priori principle, although even this is a question that 
requires further research.5 These are questions that will have to be settled elsewhere, but many chapters in this volume serve as steps towards answering them.

To conclude, the role of (neo-)Aristotelian metaphysics as I understand it is to provide a mapping of the initial limitations of any kind of rational inquiry, because without such a mapping it would be impossible to choose which of the infinitely many potential lines of research are feasible in the first place.

5 See my (2009a) for discussion on the role of the law of non-contradiction. 


\section{References}

Aristotle. Metaphysics, in The Complete Works of Aristotle, The Revised Oxford

Translation, ed. J. Barnes. 1984 Princeton University Press

Chalmers, D. J., Manley, D., and Wasserman, R. (eds.) 2009. Metametaphysics:

New Essays on the Foundations of Ontology. Oxford University Press

Correia, F. 2008. 'Ontological Dependence', Philosophy Compass 3 (5): 1013-32

Fine, K. 1994. 'Essence and Modality', J. E. Tomberlin (ed.), Philosophical Perspectives 8: Logic and Language, as the Nous Casteneda

Memorial Lecture, pp. 1-16; reprinted in P. Grim (ed.) The

Philosopher's Annual for 1994, volume 16. Stanford: CSLI

1995a. 'Ontological Dependence,' Proceedings of the Aristotelian Society 95: 269-90

2009. 'The Question of Ontology', in Chalmers, Manley, and Wasserman (eds.), pp. 157-77

Gendler, T. S. and Hawthorne J. (eds.) 2002. Conceivability and Possibility. Oxford University Press

Hendry, R. F. 2006. 'Elements, Compounds, and Other Chemical Kinds', Philosophy of Science 73: 864-75

Hofweber, T. 2005. 'A Puzzle about Ontology', Nồs 39: 256-83.

2009. 'Ambitious, Yet Modest, Metaphysics', in Chalmers, Manley, and Wasserman (eds.), pp. 260-89.

Koslicki, K. This volume. 'Essence, necessity, and explanation', pp. 187-206

Ladyman, J. and Ross, D. 2007. Every Thing Must Go: Metaphysics Naturalized. Oxford University Press

Lowe, E. J. 1998. The Possibility of Metaphysics. Oxford University Press

2006. The Four-Category Ontology: A Metaphysical Foundation for Natural

Science. Oxford: Clarendon Press

2011. 'The Rationality of Metaphysics', Synthese 178: 99-109

Quine, W. V. 1948. 'On What There Is', Review of Metaphysics 2: 21-28; reprinted in From a Logical Point of View, 2nd edn., pp. 1-19. Harvard, MA:

Harvard University Press

Schaffer, J. 2009. 'On What Ground What', in Chalmers, Manley, and Wasserman (eds.), pp. 347-383

Tahko, T. E. 2008. 'A New Definition of A Priori Knowledge: In Search of a Modal Basis', Metaphysica 9: 57-68

2009a. 'The Law of Non-Contradiction as a Metaphysical Principle', The Australasian Journal of Logic 7: 32-47

2009b. 'On the Modal Content of A Posteriori Necessities', Theoria: A Swedish Journal of Philosophy 75: 344-57 